\title{
O ensino de psiquiatria, habilidades de comunicação e atitudes no currículo integrado do curso de Medicina da Universidade Estadual de Londrina
}

\author{
The teaching of psychiatry, communication skills \\ and attitudes in the integrated curriculum of the \\ medical course at the Londrina State University.
}

Sandra Odebrecht Vargas Nunes ${ }^{1}$ Heber Odebrecht Vargas ${ }^{1}$ Marcos Liboni ${ }^{1}$

Daniel Martins Neto ${ }^{1}$

Lúcia Helena Mendonça Vargas ${ }^{1}$ Bárbara Turini ${ }^{1}$

\author{
PALAVRAS-CHAVE \\ - Psiquiatria. \\ - Comunicação. \\ - Atitudes.
}

Recebido em:16/06/2007 Reencaminhado em: 07/12/2007 Aprovado em: 20/12/2007
REVISTA BRASILEIRA DE EDUCAÇ̃̃O MÉDICA $21032(2): 210-216: 2008$

\footnotetext{
${ }^{1}$ Universidade Estadual de Londrina, Paraná, Brasil.
}

\section{A B S T R A C T}

This article describes an experience of teaching psychiatry, communication skills, and attitudes in an integrated medical curriculum at the Londrina State University. The concept map of the thematic module of mental disorders and of behavior were proposed as a strategy promoting interdisciplinary meaningful learning of Psychiatry. Psychiatric knowledge is acquired through problem-based learning. Communication skills and attitudes are taught in different ways, with patients, with simulated patients, using videos, case reports, role-playing, and work in small groups. The methods used for evaluating the knowledge in psychiatry, the communication skills and the attitudes include summative and formative assessment designed to give the student a feedback on his progress in objective structured clinical examination (Osce), portfolios, and case reports. The psychiatric knowledge, communication skills, and attitudes are assimilated depending on meaningful learning as a teaching method and on the way the students are evaluated in their medical practice. 


\section{INTRODUÇÃO}

O curso de Medicina da Universidade Estadual de Londrina (UEL) está fundamentado na pedagogia da interação, possibilitando o aperfeiçoamento contínuo de conhecimentos, habilidades e atitudes do estudante, e facilitando que este desenvolva seu próprio método de estudos, aprendendo a aprender, a selecionar criticamente os recursos educacionais mais adequados e a trabalhar em equipe ${ }^{1}$.

O currículo apresenta uma estruturação modular, que viabiliza a interdisciplinaridade, num ensino centrado nas necessidades de aprendizagem dos estudantes. Os conteúdos das ciências básicas e clínicas são desenvolvidos de forma integrada com os problemas prioritários de saúde da população e com a adoção de avaliação formativa ${ }^{1}$.

A metodologia de ensino-aprendizagem nos módulos temáticos do curso de Medicina da UEL tem um eixo metodológico que dá destaque à Aprendizagem Baseada em Problemas (ABP). Nesse sentido, é um processo muito parecido com a metodologia de pesquisa científica. O método guarda a mesma lógica: a partir de um problema, busca-se compreendê-lo, fundamentá-lo; para isto, buscam-se dados, que são analisados e discutidos; por último, são elaboradas hipóteses de solução, que devem ser colocadas em prática para serem comprovadas e validadas 2 .

Geralmente, os problemas são observados e extraídos da prática vivenciada, mas também podem ser elaborados por especialistas com base na necessidade de incorporação de determinados conceitos e princípios. A relação prática-teoria-prática deve ser priorizada, como também a integração básico-clínico e o equilíbrio entre os aspectos biopsicossociais e princípios éticos ${ }^{2}$.

Neste contexto, o ensino da psiquiatria deve ter os objetivos educacionais baseados no core currículo e divididos em: áreas de conhecimento (sintomas e síndromes psiquiátricos, aspectos psicológicos das doenças e áreas psicológicas, incluindo estigma); habilidades (relação médico-paciente, obter informação, raciocínio clínico, dar a informação, relatar, tratar, aprender e relacionar-se com a equipe); e atitudes relacionadas à prática médica, aos pacientes e familiares, e à psiquiatria como disciplina médica ${ }^{3}$.

A psiquiatria, como as demais especialidades médicas, não é ensinada como disciplina isolada. Os conhecimentos são ensinados em vários módulos temáticos, como saúde da mulher, pro- cesso de envelhecimento e emergências. Existem dois módulos em que há maior ênfase em temas específicos: percepção, consciência; emoção e problemas mentais e de comportamento. Além disso, durante o internato médico, os alunos entram em contato com a psiquiatria por meio das práticas de ambulatório, enfermaria e temas teóricos ${ }^{4}$.

Este artigo relata uma experiência de ensino de psiquiatria por meio de módulos temáticos - a exemplo do mapa conceitual do módulo de problemas mentais e de comportamento -, e sua interdisciplinaridade com os outros módulos - aquisição da habilidade de comunicação para se relacionar com pacientes, familiares, comunidade e equipe interdisciplinar, e aquisição de atitudes éticas e humanistas.

\section{A AQUISIÇÃO DO CONHECIMENTO EM PSIQUIATRIA POR MEIO DE MÓDULOS TEMÁTICOS}

Nos módulos temáticos, desenvolvem-se as atividades pedagógicas de sessões tutoriais, práticas de laboratório e discussão de casos clínicos, palestras, ensino orientado e avaliação. Estes módulos permitem capacitar os estudantes a dominar os procedimentos diagnósticos e terapêuticos necessários para a prevenção, tratamento e reabilitação dos principais problemas mentais e de comportamento.

A construção de um mapa conceitual possibilita desenvolver uma ferramenta cognitiva no processo de ensino-aprendizagem. A aprendizagem é dita significativa quando uma nova informação (conceito, idéia, percepção) adquire significado para o aprendiz por intermédio de uma ancoragem em aspectos relevantes da estrutura cognitiva preexistente do indivíduo - conceitos, idéias, percepções já existentes em sua estrutura de conhecimentos e/ou significados com determinado grau de clareza, estabilidade e diferenciação. Aprendizagem sem atribuição de significados pessoais, sem relação com o conhecimento, é mecânica e não significativa ${ }^{5}$.

A Figura 1 apresenta o mapa conceitual do módulo de problemas mentais e do comportamento, construído por meio da ferramenta cmaptools. Esse mapa mostra os conceitos-chave do módulo e suas inter-relações com os conceitos prévios e com os novos conceitos que serão aprendidos em outros módulos. 
Figura 1

Mapa conceitual do módulo de problemas mentais e de comportamento.

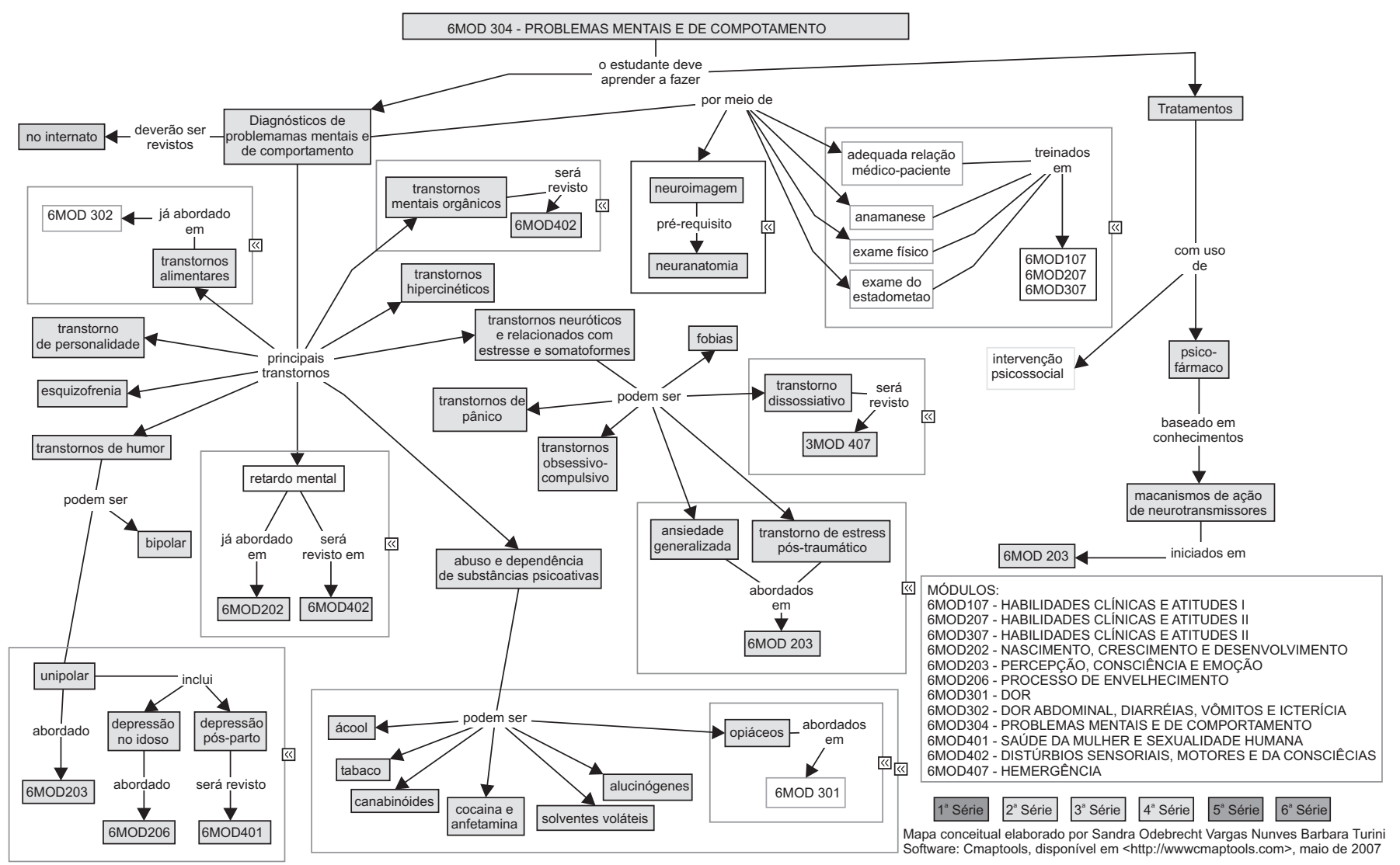

A avaliação do conhecimento é realizada por dois tipos de avaliação: formativa e somativa. A primeira ocorre durante todo o módulo e verifica se os estudantes estão adquirindo os objetivos previstos no decorrer dos módulos temáticos e habilidades, fornecendo feedback acerca do progresso e procedimentos do aluno. O método formativo determina se o aluno está atingindo cada etapa do desenvolvimento, antes de prosseguir para a etapa subseqüente. A avaliação somativa é realizada no final do módulo temático por testes de múltipla escolha e práticas ${ }^{6}$.

\section{A AQUISIÇÃO DE HABILIDADES DE COMUNIÇÇÃO SOCIAL}

Os quatro módulos de habilidades clínicas e atitudes são desenvolvidos da primeira à quarta série, anualmente, abrangendo procedimentos laboratoriais, médico-cirúrgicos, semiologia e comunicação social ${ }^{1}$.
O estímulo à aquisição de habilidades, neste contexto, pressupõe o desenvolvimento de elementos psíquicos, afetivos e cognitivos necessários à adoção de uma postura humanística no contato com o sofrimento humano. Este estímulo visa capacitar o estudante a observar aspectos sociais, culturais, psicológicos e econômicos relevantes à prática médica ${ }^{7}$.

O método de Aprendizagem Baseada em Problemas (ABP) possibilita que as escolas médicas desenvolvam nos estudantes habilidades e atitudes como trabalho em equipe, liderança de grupo, escutar, gravar, cooperação, respeito pelos pontos de vista de colegas, estudo autodirigido, uso de variados recursos e habilidades de apresentação8.

A psiquiatria participa do treinamento de habilidades em comunicação social do primeiro ao quarto ano do currículo. Enfatiza o treinamento para que o estudante de Medicina seja capaz de: executar consulta médica com os princípios da relação médi- 
co-paciente e familiares; avaliar suas próprias emoções, bem como as reações do doente frente à doença; desenvolver a capacidade de observar e ouvir; realizar entrevistas individuais nas diferentes fases do ciclo vital; informar diagnóstico, planos de tratamento e sua adesão; aprender a comunicar más notícias; conduzir manejo do paciente em situações de crise; executar o exame do estado mental.

A aquisição de habilidades em comunicação visa ensinar a importância da relação do médico com os pacientes e familiares. A interação médico-paciente é entendida como uma construção, mediada pelos contextos nos quais ocorre e pelas reações emocionais dos agentes envolvidos. Os atributos pessoais do médico e as atitudes terapêuticas desejáveis para uma boa interação médico-paciente incluem aspectos como empatia, continência e capacidades de comunicação e de conotação positiva 9 .

A aprendizagem significativa para o estudante está na maneira como interage com pacientes ou familiares que fazem parte de sua cultura, mediante entrevistas, pacientes simulados, vídeos. Todo evento de aprendizagem significativa é composto por uma relação triádica entre o educador, os materiais educativos e o estudante ${ }^{10}$.
Em habilidades de comunicação o professor poderá utilizar vários métodos de ensino, como supervisionar os atendimentos do estudante com pacientes verdadeiros ou avaliar seu atendimento com pacientes simulados, interpares, role-playing ou dramatizações, vídeos e, ao final, fornecer feedback.

As sessões de dramatização ou role-playing consistem na encenação de diferentes situações da prática médica, em que dois estudantes representam os papéis de médico e paciente, e os colegas observam o processo de comunicação aí desenvolvido, assinalando os aspectos que dificultam ou facilitam a relação médico-paciente.

Outros centros também utilizam a metodologia de pacientes simulados como estratégias de aprendizagem significativa. Nestes centros, durante o treino, o professor grava as sessões dos estudantes com os pacientes simulados e realiza avaliações ligadas ao mecanismo de realimentação (feedback), detectando as deficiências dos estudantes e possibilitando as reformulações ${ }^{11}$.

O treinamento que capacita o estudante a desenvolver as habilidades de comunicação social para adquirir os objetivos mencionados está sumarizado no Quadro 1.

\section{QUADRO 1}

Habilidades treinadas para aquisição de comunicação social

\begin{tabular}{|l|l|}
\hline \multicolumn{1}{|c|}{ Habilidades Treinadas } & \multicolumn{1}{c|}{ Métodos } \\
\hline $\begin{array}{l}\text { Habilidade interpessoal na relação médico-paciente } \\
\text { Treino com pacientes simulados, interpares, }\end{array}$ & $\begin{array}{l}\text { Entrevista com ênfase na relação médico-paciente. Motivar para a } \\
\text { comunicação não-verbal (olhar, postura, escuta ativa, toques } \\
\text { adequados, empatia), comunicação verbal (linguagem acessível) e } \\
\text { compromisso com privacidade e sigilo }\end{array}$ \\
\hline Habilidades para obter a informação & $\begin{array}{l}\text { Entrevistas, técnicas de dramatização ou role-playing, exame do } \\
\text { estado mental e história de vida do paciente } \\
\text { Vídeos com depoimentos de pacientes }\end{array}$ \\
\hline Habilidade para avaliar a informação & $\begin{array}{l}\text { Prática de entrevista para o raciocínio clínico e diagnóstico } \\
\text { abrangente }\end{array}$ \\
\hline Habilidade para dar a informação & $\begin{array}{l}\text { Entrevistas com pacientes com doenças crônicas, incapacitantes e } \\
\text { terminais; entrevistas com familiares e médicos destes pacientes }\end{array}$ \\
\hline Habilidade de adesão a plano de tratamento & $\begin{array}{l}\text { Entrevistas para avaliar a adesão ao tratamento medicamentoso e } \\
\text { não medicamentoso } \\
\text { Técnicas de dramatização }\end{array}$ \\
\hline Habilidade de aprender & Motivação para auto-aprendizagem \\
\hline
\end{tabular}


As avaliações dos módulos de habilidades clínicas e atitudes são realizadas com base no desempenho clínico no Exame Clínico Estruturado por Objetivo (Osce), relatórios de casos clínicos e portfolios (avaliação formativa). Todas essas técnicas permitem acompanhar o progresso e corrigir as debilidades do estudante.

\section{AQUISIÇÃO DE ATITUDES}

Nos módulos de habilidades clínicas e atitudes prepara-se o estudante para exercer a medicina com postura ética e visão humanística para com o paciente, a família e a comunidade, e também com os demais membros de equipes multiprofissionais. Esses módulos capacitam o estudante a observar os aspectos sociais, culturais, psicológicos e econômicos, relevantes para a prática médica.

As atitudes adquiridas na psiquiatria são as mesmas para todas as disciplinas do curso de Medicina, pois o projeto político-pedagógico do curso integrado de Medicina da UEL estimula a compreensão do paciente como ser biopsicossocial e a prática da educação permanente.

As atitudes não são expressas verbalmente, mas internalizadas por treinamento com estratégias facilitadoras de uma apren- dizagem significativa nos módulos temáticos interdisciplinares, módulos de habilidades clínicas e atitudes, e no internato médico de dois anos.

Exercer a medicina com postura ética e desenvolver uma identidade médica são passos essenciais para se tornar um bom médico. Líderes em educação médica devem criar novos modelos de treinamento para complementar os métodos tradicionais de aprendizagem em educação médica. Sugere-se, ainda, a ênfase em disciplinas centradas no paciente ${ }^{12}$.

A preocupação com o desenvolvimento moral dos estudantes deve ser um foco de importância para a aquisição e desenvolvimento de habilidades na relação médico-paciente, no contexto tanto da psiquiatria, como da medicina. Os pressupostos éticos e bioéticos da relação impõem ao futuro profissional um diferencial sobre não só o que fazer, mas como e se é possível fazer algo pelo paciente ou pelas populações com as quais está em contato em seu cotidiano ${ }^{13}$.

O treinamento para o estudante desenvolver habilidades de atitudes está sumarizado no Quadro 2.

\section{QUADRO 2}

Habilidades treinadas para aquisição de atitudes

\begin{tabular}{|c|c|}
\hline Atitudes Treinadas & Métodos \\
\hline Postura ética e humanística & $\begin{array}{l}\text { Avaliação formativa nos grupos tutoriais, habilidades clínicas e } \\
\text { atitudes no internato }\end{array}$ \\
\hline $\begin{array}{l}\text { Atitudes em relação aos pacientes, familiares e } \\
\text { comunidade }\end{array}$ & $\begin{array}{l}\text { Treinar a respeitar o paciente e familiares e compreender seus } \\
\text { sentimentos por meio de entrevistas } \\
\text { Entrevistas e vídeos para reconhecer atitudes adequadas e } \\
\text { inadequadas dos médicos frente ao paciente e familiares } \\
\text { Exame Clínico Estruturado por Objetivo (Osce), realizado do primeiro } \\
\text { ao quarto ano, com pacientes simulados }\end{array}$ \\
\hline $\begin{array}{l}\text { Atitudes para compreender o paciente como ser } \\
\text { biopsicossocial }\end{array}$ & $\begin{array}{l}\text { Problemas dos tutoriais, entrevistas e prática de atendimento ao } \\
\text { paciente }\end{array}$ \\
\hline Atitude crítica e reflexiva & Auto-avaliação, avaliação interpares, avaliação do tutor \\
\hline Atuar em equipe multiprofissional e em grupos & $\begin{array}{l}\text { Atividades e discussões em pequenos grupos, relacionando-se com os } \\
\text { demais membros do grupo em bases éticas }\end{array}$ \\
\hline
\end{tabular}


A aquisição de atitudes, conhecimentos e habilidades constitui um processo contínuo ao longo da vida de cada indivíduo. Portanto, os estudantes do curso de Medicina da UEL são encorajados a definir seus objetivos de aprendizagem e a assumir a responsabilidade de avaliar seus progressos pessoais ${ }^{1}$.

A adoção de um sistema de avaliação não apenas cognitivo, mas um sistema de avaliação formativa e de desempenho clínico, no Exame Clínico Estruturado por Objetivo (Osce), com feedback para o estudante, propicia que este internalize atitudes.

As competências profissionais desejadas para o médico formado no projeto político-pedagógico da UEL incluem, entre outras, o exercício da medicina com postura ética e visão humanística do paciente, de sua família e da comunidade. As competências profissionais ensinadas em escolas médicas devem incluir o uso da habilidade em comunicação, conhecimentos validados cientificamente, habilidades técnicas, raciocínio clínico, domínio moral e afetivo, e reflexão, na prática diária, em benefício do indivíduo e da comunidade a que serve ${ }^{14}$.

Na UEL, a mudança não se limitou ao método de ensino-aprendizagem, mas abrangeu a filosofia educacional, que incluiu a integração vertical e horizontal de disciplinas e a avaliação somativa e formativa do estudante ${ }^{15}$. A avaliação formativa também tem como objetivo o mecanismo de realimentação, pois permite ao professor detectar e identificar deficiências na forma de ensinar, possibilitando reformulações em seu trabalho didático a fim de aperfeiçoá-lo quando ainda em curso. Estas modalidades de avaliação permitem que o estudante alcance o objetivo desejado ${ }^{1}$.

\section{CONSIDERAÇÕES FINAIS}

Este estudo se insere numa discussão do ensino de psiquiatria, utilizando a metodologia de Aprendizagem Baseada em Problemas. O mapa conceitual do módulo de problemas mentais e do comportamento foi proposto como uma estratégia facilitadora para uma aprendizagem significativa, isto é, um diagrama conceitual que envolve os principais conceitos de psiquiatria e suas relações.

O treinamento proposto para habilidades de comunicação e atitudes pressupõe o desenvolvimento dos elementos psíquicos, afetivos e cognitivos necessários ao desenvolvimento de uma postura humanística no contato com o sofrimento humano. As formas de desenvolvimento das habilidades ocorrem de diferentes modos: com pacientes verdadeiros, pacientes simulados, interpares, role-playing ou dramatizações, vídeos e relatos de casos clínicos.
No currículo integrado do curso de Medicina da UEL, composto por uma metodologia ativa de ensino-aprendizagem, avaliação somativa e formativa, ênfase em módulos interdisciplinares - exemplificado pelo mapa conceitual do módulo temático de problemas mentais e do comportamento-, está sendo desenvolvido e internalizado o conhecimento em psiquiatria, habilidades de comunicação e atitudes.

\section{REFERÊNCIAS}

1. Universidade Estadual de Londrina. Colegiado do Curso de Medicina. Atos Legais de Consolidação do Currículo Integrado. Londrina; 2006.

2. Universidade Estadual de Londrina. Colegiado do Curso de Medicina. Projeto Político-Pedagógico do Curso de Medicina. Londrina; 2004.

3. World Psychiatric Association. Core Curriculum in psychiatry for medical students. Med Educ 1999; 33:204-11.

4. Nunes SOV, Vargas H, Costa Benquerer F, Bueno C, Celeste L, Oliveira R, et al. O Ensino da Psiquiatria na Graduação de Medicina pelo Método de Aprendizagem Baseada em Problemas (P.B.L.). Psiquiatria Biológica 2002; 10(1): 23-26.

5. Moreira M A. Mapas Conceituais e Aprendizagem Significativa. [on line].[capturado 17 jun. 2007] Disponível em: http:/ / www.if.ufrgs.br/ moreira/mapasport.pdf

6. Universidade Estadual de Londrina. Colegiado do Curso de Medicina Sistema Integrado de Avaliação do Curso de Medicina da UEL SIAMed. Londrina; 2003.

7. Siqueira JE. A evolução científica e tecnológica, o aumento dos custos em saúde e a questão da universalidade do acesso. Bioética 1997; 5(1): 41-8.

8. Wood D. ABC of learning and teaching in medicine. Problem Based learning. BMJ 2003; 326: 328-30.

9. Soar Filho EJ. A interação médico-paciente. Rev Assoc Med Bras 1998;44(1):35-42.

10. Pires M, Veit E. Tecnologias de informação e Comunicação para ampliar e motivar o aprendizado de Física no Ensino Médio. Revista Brasileira de Ensino de Física 2006; 28(2): 241-8.

11. Sharp P, Pearce K, Konen J, Knudson M. Using standardized patient instructors to teach health promotion interviewing skill. Fam Med 1996; 28:103-6.

12. Roberts L, Warner T, Hammond G, Geppert C, Heinrich T. Becoming a good doctor: perceived need for ethics training focused on practical and professional development topics. Acad Psychiatry 2005; 29(3): 301-9. 
13. Liboni M. A Estrutura da Sensibilidade Moral dos Psiquiatras do Brasil. São Paulo; 2005. Doutorado [Tese] - Universidade de São Paulo.

14. Epstein R, Humbert E. Defining and assessing professional competence. JAMA 2002; 287(9):226-35.

15. Cyrino E, Toralles-Pereira ML. Trabalhando com estratégias de ensino-aprendizagem por descoberta na área da saúde: a problematização e a aprendizagem baseada em problemas. Cad Saude Publica 2004; 20(3):780-8.

\section{CONFLITOS DE INTERESSE}

Declarou não haver

\section{ENDEREÇO PARA CORRESPONDÊNCIA}

Sandra Odebrecht Vargas Nunes

Av. Adhemar de Barros, 625

85050-190 - Londrina - PR

E-mail: sandranunes@sercomtel.com.br 